\title{
CUBIC MOMENTS OF FOURIER COEFFICIENTS AND PAIRS OF DIAGONAL QUARTIC FORMS
}

\author{
JÖRG BRÜDERN AND TREVOR D. WOOLEY
}

\begin{abstract}
We establish the non-singular Hasse Principle for pairs of diagonal quartic equations in 22 or more variables. Our methods involve the estimation of a certain entangled two-dimensional $21^{\text {st }}$ moment of quartic smooth Weyl sums via a novel cubic moment of Fourier coefficients.
\end{abstract}

\section{INTRODUCTION}

A consideration of disjoint systems of diagonal Diophantine equations lends credibility to the guiding principle that the number of variables required to solve a system should in general grow in proportion to the number of its equations. That such systems are no harder to analyse is made evident in an elegant paper of Cook [6]. There is remarkably little work available in which systems of entangled equations have been successfully analysed in circumstances where the average number of variables per equation is smaller than that accessible for a single equation. These investigations have been limited almost exclusively to equations of degree at most three, and exploit the interaction between component equations by means of restricted moments of Fourier coefficients of unconventional type (see [3, 4, 5]). In this paper we add to this small stock of examples, analysing pairs of diagonal quartic forms through a novel cubic moment of certain Fourier coefficients of arithmetic origin.

In order to describe our results, we introduce some notation. When $s$ is a natural number, let $a_{j}, b_{j}(1 \leqslant j \leqslant s)$ be fixed rational integers, and consider the pair of simultaneous diagonal quartic equations

$$
a_{1} x_{1}^{4}+a_{2} x_{2}^{4}+\ldots+a_{s} x_{s}^{4}=b_{1} x_{1}^{4}+b_{2} x_{2}^{4}+\ldots+b_{s} x_{s}^{4}=0 .
$$

Given a positive number $P$, we denote by $\mathcal{N}(P)$ the number of integral solutions $\mathbf{x}$ of (1.1) with $\left|x_{j}\right| \leqslant P(1 \leqslant j \leqslant s)$.

Theorem 1.1. Let $s$ be a natural number with $s \geqslant 22$. Suppose that $a_{i}, b_{i} \in \mathbb{Z}$ $(1 \leqslant i \leqslant s)$ satisfy the condition that for any pair $(c, d) \in \mathbb{Z}^{2} \backslash\{(0,0)\}$, at least $s-7$ of the numbers $c a_{j}+d b_{j}(1 \leqslant j \leqslant s)$ are non-zero. Then provided that the system (1.1) has non-singular real and p-adic solutions for each prime number $p$, one has $\mathcal{N}(P) \gg P^{s-8}$.

2010 Mathematics Subject Classification. 11D72, 11P55, 11E76.

Key words and phrases. Quartic Diophantine equations, Hardy-Littlewood method.

The authors are grateful to the Hausdorff Research Institute for Mathematics in Bonn and the Heilbronn Institute for Mathematics Research in Bristol for excellent working conditions that made the writing of this paper feasible. 
We pause at this point to discuss the various hypotheses of this theorem. First, the application of conventional technology (see [1, 12]) has the potential to establish a conclusion analogous to Theorem 1.1 only for $s \geqslant 24$. Whilst our conclusion saves two variables over this bound, one may safely conjecture that subject to appropriate rank conditions the constraint $s \geqslant 17$ should suffice. When $s \geqslant 21$, it follows from work of Godinho [8] that the system (1.1) has non-zero $p$-adic solutions whenever $p>73$. Theorem 1.1 consequently implies a Hasse principle for pairs of equations of the shape (1.1) in a rather strong form. By combining Godinho's work with that of Poehler [10], meanwhile, one finds that the $p$-adic solubility of the system (1.1) is assured for $s \geqslant 49$.

The most novel feature of our proof of Theorem 1.1 involves a consideration of a suitable cubic moment of certain Fourier coefficients. For a continuous function $H: \mathbb{R} \rightarrow[0, \infty)$ of period 1 , let $c(n)=\int_{0}^{1} H(\alpha) e(-n \alpha) \mathrm{d} \alpha$, where as usual we write $e(z)$ for $e^{2 \pi i z}$. It transpires that one may relate the moment

$$
\sum_{n \in \mathbb{Z}}|c(n)|^{3}
$$

to the correlation

$$
\int_{0}^{1} \int_{0}^{1} H(\alpha) H(\beta) H(\alpha+\beta) \mathrm{d} \alpha \mathrm{d} \beta .
$$

We shall take $H(\alpha)=\left|\sum_{x \in \mathcal{A}} e\left(\alpha x^{4}\right)\right|^{7}$, in which $\mathcal{A}$ is a suitable set of smooth numbers. In this case, one may control the moment (1.2) by means of the large values estimates for Fourier coefficients provided in [9]. The mean value (1.3) may be viewed as an entangled $21^{\text {st }}$-moment of smooth Weyl sums, and it is this that plays the leading role in our application of the Hardy-Littlewood (circle) method. We remark that with $H(\alpha)$ defined as above, the Fourier coefficient $c(n)$ has no direct arithmetic interpretation, despite its arithmetic origin. Indeed, this coefficient may be non-zero for arbitrarily large $n$, a complication reflected in technical difficulties that we encounter when relating (1.2) to (1.3) in $§ 3$. Although in principle our methods are applicable in wide generality, it would appear that in light of currently available mean value estimates for Weyl sums, new ideas are required for their application to Diophantine problems of higher degree.

Following some preliminary discussion of smooth Weyl sums, we announce the key $21^{\mathrm{st}}$-moment estimate in $\S 2$. The aforementioned cubic moment of Fourier coefficients is analysed in $\S 3$, and then estimated by means of large values estimates in $\S 4$. In $\S 5$ we shift our focus to preparations for the application of the circle method, tackling the minor arcs in the Hardy-Littlewood dissection in $\S 6$, and concluding with the major arc analysis in $\S 7$.

Our basic parameter is $P$, a sufficiently large positive number. In this paper, implicit constants in Vinogradov's notation $\ll$ and $\gg$ may depend on $s$ and $\varepsilon$, as well as ambient coefficients such as $\mathbf{a}$ and $\mathbf{b}$. Whenever $\varepsilon$ appears in a statement, either implicitly or explicitly, we assert that the statement holds for each $\varepsilon>0$. 


\section{A $21^{\text {st }}$-MOMENT OF BIQUADRATIC WEYL SUMS}

In this section we introduce the novel ingredients utilised in our application of the circle method. Before announcing these unconventional mean value estimates, however, we must introduce some notation. When $P$ and $R$ are real numbers with $1 \leqslant R \leqslant P$, we define the set of smooth numbers $\mathcal{A}(P, R)$ by

$$
\mathcal{A}(P, R)=\{n \in \mathbb{Z} \cap[1, P]: p \text { prime and } p \mid n \Rightarrow p \leqslant R\} .
$$

We then define the smooth Weyl sum $h(\alpha)=h(\alpha ; P, R)$ by

$$
h(\alpha ; P, R)=\sum_{x \in \mathcal{A}(P, R)} e\left(\alpha x^{4}\right) .
$$

It is convenient to refer to an exponent $\Delta_{t}$ as admissible if there exists a positive number $\eta$ such that, whenever $1 \leqslant R \leqslant P^{\eta}$, one has

$$
\int_{0}^{1}|h(\alpha ; P, R)|^{t} \mathrm{~d} \alpha \ll P^{t-4+\Delta_{t}} .
$$

Lemma 2.1. The exponents $\Delta_{7}=0.849408, \Delta_{10}=0.213431$ and $\Delta_{12}=0$ are admissible.

Proof. The desired conclusion follows from [2, Theorem 2] and the discussion surrounding the table of exponents on [2, page 393]. As explained in the proof of [2, Lemma 2.3], the upper bound (2.1) when $t=12$ is a consequence of [12, Lemma 5.2].

Henceforth, we fix $R=P^{\eta}$ with $\eta>0$ chosen in accordance with Lemma 2.1 and the upper bounds (2.1). When $a, b, c, d \in \mathbb{Z}$, we define the integral

$$
I(a, b, c, d)=\int_{0}^{1} \int_{0}^{1}|h(a \alpha) h(b \beta) h(c \alpha+d \beta)|^{7} \mathrm{~d} \alpha \mathrm{d} \beta .
$$

Our goal in $\S \S 3$ and 4 is the proof of the following upper bound for this integral.

Theorem 2.2. Suppose that $a, b, c, d$ are non-zero integers. Then one has

$$
I(a, b, c, d) \ll P^{13+\frac{1}{2} \Delta_{10}+\varepsilon} .
$$

Previous authors would disentangle the mixed moment (2.2) via Hölder's inequality to obtain an estimate of the shape

$$
I(a, b, c, d) \ll\left(\int_{0}^{1}|h(A \alpha)|^{21 / 2} \mathrm{~d} \alpha\right)^{2} \ll P^{13+\frac{3}{2} \Delta_{10}} .
$$

The superiority of our new estimate is self-evident. We direct the curious reader to the explanation following the statement of [3, Theorem 3] for a related discussion. By following the argument of the proof of [3, Theorem 4], presented in $\S 4$ of the latter source, one obtains from Theorem 2.2 a superficially more general conclusion of similar type.

Theorem 2.3. Suppose that $c_{i}, d_{i}(1 \leqslant i \leqslant 3)$ are integers satisfying

$$
\left(c_{1} d_{2}-c_{2} d_{1}\right)\left(c_{1} d_{3}-c_{3} d_{1}\right)\left(c_{2} d_{3}-c_{3} d_{2}\right) \neq 0 \text {. }
$$


Write $\Lambda_{j}=c_{j} \alpha+d_{j} \beta(j=1,2,3)$. Then whenever $1 \leqslant R \leqslant P^{\eta}$, one has

$$
\int_{0}^{1} \int_{0}^{1}\left|h\left(\Lambda_{1}\right) h\left(\Lambda_{2}\right) h\left(\Lambda_{3}\right)\right|^{7} \mathrm{~d} \alpha \mathrm{d} \beta \ll P^{13+\frac{1}{2} \Delta_{10}+\varepsilon} .
$$

\section{Cubic moments of certain Fourier coefficients}

An inspection of the mean value (2.2) suggests that Fourier coefficients associated with $|h(\alpha)|^{7}$ should play a prominent role in its estimation. The absence of any direct arithmetic interpretation forces us, however, to indulge in a far more detailed discussion of these Fourier coefficients than would ordinarily be the case. Define

$$
\psi(n)=\int_{0}^{1}|h(\alpha)|^{7} e(-n \alpha) \mathrm{d} \alpha .
$$

Lemma 3.1. The Fourier expansion

$$
|h(\alpha)|^{7}=\sum_{n \in \mathbb{Z}} \psi(n) e(n \alpha)
$$

is uniformly convergent in $\alpha$, and one has $\psi(n) \ll P^{15} / n^{2}$.

Proof. We begin by observing that $h(\alpha)$ is an analytic function, and hence $|h(\alpha)|^{7}$ is a real valued function that is twice continuously differentiable. Note that when $h(\alpha)=0$, then the first two derivatives of $|h(\alpha)|^{7}$ are also 0. By writing $|h(\alpha)|^{7}=h(\alpha)^{7 / 2} h(-\alpha)^{7 / 2}$ when $h(\alpha) \neq 0$, and noting that

$$
h^{\prime}(\alpha)=2 \pi i \sum_{x \in \mathcal{A}(P, R)} x^{4} e\left(\alpha x^{4}\right) \ll P^{5}
$$

and

$$
h^{\prime \prime}(\alpha)=(2 \pi i)^{2} \sum_{x \in \mathcal{A}(P, R)} x^{8} e\left(\alpha x^{4}\right) \ll P^{9},
$$

it follows that, uniformly in $\alpha$, one has $\frac{\mathrm{d}^{2}}{\mathrm{~d} \alpha^{2}}|h(\alpha)|^{7} \ll P^{15}$. Consequently, by integrating by parts, one deduces that

$$
\int_{0}^{1}|h(\alpha)|^{7} e(-n \alpha) \mathrm{d} \alpha=\int_{0}^{1} \frac{e(-n \alpha)}{(2 \pi i n)^{2}} \frac{\mathrm{d}^{2}}{\mathrm{~d} \alpha^{2}}|h(\alpha)|^{7} \mathrm{~d} \alpha \ll \frac{P^{15}}{n^{2}} .
$$

Finally, since $|h(\alpha)|^{7}$ is differentiable and the series on the right hand side of (3.2) is absolutely convergent, one finds from [7, Theorem 1.4.2] or [16, Theorem 8.14, page 60], for example, that the Fourier expansion (3.2) converges to $|h(\alpha)|^{7}$ uniformly in $\alpha$. This completes the proof of the lemma.

This lemma allows us to replace the mean value (2.2) by a cubic moment of $\psi(n)$, truncated both in terms of $n$ and the magnitude of $\psi(n)$.

Lemma 3.2. When $a, b, c, d$ are non-zero integers, one has

$$
I(a, b, c, d) \ll P^{9}+\sum_{\substack{|n|<P^{9} \\|\psi(n)|>1}}|\psi(n)|^{3} .
$$


Proof. Our initial step is to accommodate non-zero integral coefficients $l$ by extracting from (3.2) the relation

$$
|h(l \alpha)|^{7}=\sum_{n \in \mathbb{Z}} \psi(n) e(n l \alpha)=\sum_{m \in \mathbb{Z}} \psi_{l}(m) e(m \alpha),
$$

where

$$
\psi_{l}(m)= \begin{cases}\psi(m / l), & \text { when } l \mid m, \\ 0, & \text { otherwise. }\end{cases}
$$

Using the uniform convergence of the Fourier expansions of $|h(a \alpha)|^{7},|h(b \beta)|^{7}$ and $|h(c \alpha+d \beta)|^{7}$, one finds from (2.2) that $I(a, b, c, d)$ is equal to

$$
\sum_{n_{1}, n_{2}, n_{3} \in \mathbb{Z}} \int_{0}^{1} \int_{0}^{1} \psi_{a}\left(n_{1}\right) \psi_{b}\left(n_{2}\right) \psi\left(n_{3}\right) e\left(n_{1} \alpha+n_{2} \beta-n_{3}(c \alpha+d \beta)\right) \mathrm{d} \alpha \mathrm{d} \beta .
$$

By orthogonality, the double integral here can be non-zero only when $n_{1}=c n_{3}$ and $n_{2}=d n_{3}$, whence

$$
I(a, b, c, d)=\sum_{n \in \mathbb{Z}} \psi_{a}(c n) \psi_{b}(d n) \psi(n) .
$$

Thus, on noting that (3.3) delivers the estimate

$$
\sum_{n \in \mathbb{Z}}\left|\psi_{a}(c n)\right|^{3} \leqslant \sum_{m \in \mathbb{Z}}\left|\psi_{a}(m)\right|^{3}=\sum_{k \in \mathbb{Z}}|\psi(k)|^{3},
$$

we deduce by Hölder's inequality and symmetry that

$$
I(a, b, c, d) \leqslant \sum_{n \in \mathbb{Z}}|\psi(n)|^{3} .
$$

We therefore conclude from Lemma 3.1 that

$$
I(a, b, c, d) \ll \sum_{|n| \leqslant P^{9}}|\psi(n)|^{3}+\sum_{|n|>P^{9}}\left(P^{15} / n^{2}\right)^{3}=\sum_{\substack{|n| \leqslant P^{9} \\|\psi(n)|>1}}|\psi(n)|^{3}+O\left(P^{9}\right),
$$

and the proof of the lemma is complete.

A dyadic dissection simplifies the discussion to come. When $T>0$, write

$$
M(T)=\sum_{\substack{|n| \leqslant P^{9} \\ T<|\psi(n)| \leqslant 2 T}}|\psi(n)|^{3} .
$$

By applying the triangle inequality to (3.1), the estimates available from Lemma 2.1 furnish the bound $\psi(n) \leqslant \psi(0) \ll P^{3+\Delta_{7}}$, and hence

$$
\sum_{\substack{|n| \leqslant P^{9} \\|\psi(n)|>1}}|\psi(n)|^{3} \leqslant \sum_{\substack{l=0 \\ 2^{l} \leqslant P^{3+\Delta_{7}} \log P}}^{\infty} M\left(2^{l}\right) .
$$

Consequently, for some positive number $T$ with $1 \leqslant T \leqslant P^{3+\Delta_{7}} \log P$, we may conclude from Lemma 3.2 that

$$
I(a, b, c, d) \ll P^{9}+(\log P) M(T) .
$$




\section{The ARITHMETIC HARMONIC ANALYSIS}

The moment has come to deliver the proof of Theorem 2.2. We may suppose that $\eta>0$ is small enough that the estimates implicit in (2.1) hold. We bound $M(T)$ when $1 \leqslant T \leqslant P^{3+\Delta_{7}} \log P$. Define $\mathcal{Z}$ to be the set of integers $n$ with $|n| \leqslant P^{9}$ such that $T<|\psi(n)| \leqslant 2 T$, and write $Z=\operatorname{card}(\mathcal{Z})$. For each $n \in \mathcal{Z}$, we take $\omega_{n}=1$ when $\psi(n)>0$, and $\omega_{n}=-1$ when $\psi(n)<0$, and then put

$$
K(\alpha)=\sum_{n \in \mathcal{Z}} \omega_{n} e(-n \alpha) .
$$

Then we find from (3.1) that

$$
\int_{0}^{1}|h(\alpha)|^{7} K(\alpha) \mathrm{d} \alpha=\sum_{n \in \mathcal{Z}} \omega_{n} \int_{0}^{1}|h(\alpha)|^{7} e(-n \alpha) \mathrm{d} \alpha=\sum_{n \in \mathcal{Z}}|\psi(n)|>T Z .
$$

We bound $M(T)$ by estimating the integral on the left hand side of (4.1), controlling the frequency of large Fourier coefficients $\psi(n)$.

Before proceeding further, we recall that the estimate

$$
\int_{0}^{1}\left|h(\alpha)^{4} K(\alpha)^{2}\right| \mathrm{d} \alpha \ll P^{3} Z+P^{2+\varepsilon} Z^{3 / 2}
$$

is an immediate consequence of [9, Lemma 2.1].

Lemma 4.1. One has the estimates

$$
Z \ll P^{\frac{28}{3}+\frac{1}{3} \Delta_{10}} T^{-2}+P^{13+\frac{1}{2} \Delta_{10}+\varepsilon} T^{-3}
$$

and

$$
Z \ll P^{9+\Delta_{10}} T^{-2}+P^{16+2 \Delta_{10}+\varepsilon} T^{-4} .
$$

Proof. An application of Hölder's inequality shows that

$$
\begin{aligned}
\int_{0}^{1}|h(\alpha)|^{7} K(\alpha) \mathrm{d} \alpha \leqslant & \left(\int_{0}^{1}\left|h(\alpha)^{4} K(\alpha)^{2}\right| \mathrm{d} \alpha\right)^{1 / 3}\left(\int_{0}^{1}|K(\alpha)|^{2} \mathrm{~d} \alpha\right)^{1 / 6} \\
& \times\left(\int_{0}^{1}|h(\alpha)|^{10} \mathrm{~d} \alpha\right)^{1 / 6}\left(\int_{0}^{1}|h(\alpha)|^{12} \mathrm{~d} \alpha\right)^{1 / 3} .
\end{aligned}
$$

Recalling Lemma 2.1, Parseval's identity and (4.2), we deduce from (4.1) that

$$
\begin{aligned}
T Z & \ll\left(P^{3} Z+P^{2+\varepsilon} Z^{3 / 2}\right)^{1 / 3}(Z)^{1 / 6}\left(P^{6+\Delta_{10}}\right)^{1 / 6}\left(P^{8}\right)^{1 / 3} \\
& \ll P^{\frac{14}{3}+\frac{1}{6} \Delta_{10}} Z^{1 / 2}+P^{\frac{13}{3}+\frac{1}{6} \Delta_{10}+\varepsilon} Z^{2 / 3},
\end{aligned}
$$

and the first of the claimed estimates follows by disentangling this inequality.

On the other hand, also by Hölder's inequality, one has similarly

$$
\begin{aligned}
\int_{0}^{1}|h(\alpha)|^{7} K(\alpha) \mathrm{d} \alpha & \leqslant\left(\int_{0}^{1}\left|h(\alpha)^{4} K(\alpha)^{2}\right| \mathrm{d} \alpha\right)^{1 / 2}\left(\int_{0}^{1}|h(\alpha)|^{10} \mathrm{~d} \alpha\right)^{1 / 2} \\
& \ll\left(P^{3} Z+P^{2+\varepsilon} Z^{3 / 2}\right)^{1 / 2}\left(P^{6+\Delta_{10}}\right)^{1 / 2},
\end{aligned}
$$

whence

$$
T Z \ll P^{\frac{9}{2}+\frac{1}{2} \Delta_{10}} Z^{1 / 2}+P^{4+\frac{1}{2} \Delta_{10}+\varepsilon} Z^{3 / 4},
$$

and the second conclusion follows by further disentangling. 
We are now equipped to establish Theorem 2.2. On the one hand, if one has $T \leqslant P^{3+\frac{3}{2} \Delta_{10}}$, then one finds from the first estimate of Lemma 4.1 that

$$
M(T) \ll Z T^{3} \ll P^{\frac{28}{3}+\frac{1}{3} \Delta_{10}} T+P^{13+\frac{1}{2} \Delta_{10}+\varepsilon} \ll P^{13+\frac{1}{2} \Delta_{10}+\varepsilon} .
$$

On the other hand, when $P^{3+\frac{3}{2} \Delta_{10}}<T \leqslant P^{3+\Delta_{7}} \log P$, one finds instead from the second estimate of Lemma 4.1 that

$$
Z T^{3} \ll P^{9+\Delta_{10}} T+P^{16+2 \Delta_{10}+\varepsilon} T^{-1} \ll P^{12+\Delta_{7}+\Delta_{10}+\varepsilon}+P^{13+\frac{1}{2} \Delta_{10}+\varepsilon} .
$$

Thus, on recalling (3.4), we deduce that

$$
I(a, b, c, d) \ll P^{9}+(\log P) P^{13+\frac{1}{2} \Delta_{10}+\varepsilon} \ll P^{13+\frac{1}{2} \Delta_{10}+2 \varepsilon} .
$$

This concludes the proof of Theorem 2.2 .

\section{Preparations For the CirCle method}

We suppose that the hypotheses of the statement of Theorem 1.1 are satisfied, so in particular $s \geqslant 22$. With the pairs $\left(a_{j}, b_{j}\right) \in \mathbb{Z}^{2} \backslash\{(0,0)\}$, we associate the binary forms

$$
\Lambda_{j}=a_{j} \alpha+b_{j} \beta \quad(1 \leqslant j \leqslant s),
$$

and the two linear forms $L_{1}(\boldsymbol{\theta})$ and $L_{2}(\boldsymbol{\theta})$ defined for $\boldsymbol{\theta} \in \mathbb{R}^{s}$ by

$$
L_{1}(\boldsymbol{\theta})=\sum_{j=1}^{s} a_{j} \theta_{j} \quad \text { and } \quad L_{2}(\boldsymbol{\theta})=\sum_{j=1}^{s} b_{j} \theta_{j} .
$$

We describe two forms $\Lambda_{i}$ and $\Lambda_{j}$ as equivalent when there exists a non-zero rational number $\lambda$ with $\Lambda_{i}=\lambda \Lambda_{j}$. This notion defines an equivalence relation, and we refer to the number of elements in the equivalence class containing the form $\Lambda_{j}$ as its multiplicity.

The hypotheses of Theorem 1.1 ensure that there is a non-singular real solution of the system (1.1). By invoking homogeneity, therefore, one finds that there exists a real solution $\mathbf{x}=\boldsymbol{\theta}$ in $[0,1)^{s}$ satisfying the property that for some indices $i$ and $j$ with $1 \leqslant i<j \leqslant s$, one has

$$
\operatorname{det}\left(\begin{array}{cc}
4 a_{i} \theta_{i}^{3} & 4 a_{j} \theta_{j}^{3} \\
4 b_{i} \theta_{i}^{3} & 4 b_{j} \theta_{j}^{3}
\end{array}\right) \neq 0 .
$$

By relabelling variables if necessary, there is no loss of generality in supposing that $i=1$ and $j=2$, and by taking suitable linearly independent linear combinations of the equations comprising (1.1), we may suppose further that $a_{2}=b_{1}=0$ and $a_{1} b_{2} \neq 0$. Thus, since $\boldsymbol{\theta}$ is a non-singular solution, we have $\theta_{1} \theta_{2} \neq 0$, and then there is no loss of generality in supposing also that $\theta_{1}>0$ and $\theta_{2}>0$. An application of the inverse function theorem consequently confirms that whenever $\delta>0$ is sufficiently small, the simultaneous equations

$$
a_{1} x_{1}^{4}=-\sum_{i=3}^{s} a_{i}\left(\theta_{i}+\delta\right)^{4} \quad \text { and } \quad b_{2} x_{2}^{4}=-\sum_{i=3}^{s} b_{i}\left(\theta_{i}+\delta\right)^{4}
$$

remain soluble for $x_{1}$ and $x_{2}$, with $x_{1}>0$ and $x_{2}>0$. In this way we see that the system (1.1) possesses a non-singular real solution $\boldsymbol{\theta}$ satisfying $\boldsymbol{\theta} \in(0,1)^{s}$. 
For any pair $(c, d) \in \mathbb{Z}^{2} \backslash\{(0,0)\}$, the linear form $c L_{1}(\boldsymbol{\xi})+d L_{2}(\boldsymbol{\xi})$ necessarily possesses at least $s-7$ non-zero coefficients. By choosing an appropriate subset $\mathcal{S}$ of $\{2, \ldots, s\}$ with $\operatorname{card}(\mathcal{S})=21$, therefore, we may ensure that the forms $\Lambda_{j}$ with $j \in \mathcal{S}$ have multiplicity at most 7 . Suppose that these 21 forms fall into $t$ equivalence classes, and that the multiplicities of the representatives of these classes are $r_{1}, \ldots, r_{t}$. Then we may suppose that

$$
7 \geqslant r_{1} \geqslant r_{2} \geqslant \ldots \geqslant r_{t} \text { and } r_{1}+\ldots+r_{t}=21,
$$

and hence also that $t \geqslant 3$. We relabel variables in (1.1), and likewise in (5.1) and (5.2), so that $\mathcal{S}$ becomes $\{2, \ldots, 22\}$, and for $1 \leqslant i \leqslant t$ the linear form $\Lambda_{i+1}$ is in the $i$ th equivalence class counted by $r_{i}$. We now fix a non-singular real solution $\boldsymbol{\theta} \in(0,1)^{s}$ of (1.1) and a real number $\delta$ with $0<\delta<\theta_{1}$. In addition, we fix $\eta>0$ to be sufficiently small in the context of Lemma 2.1.

Next define the generating functions

$$
g(\alpha)=\sum_{\delta P<x \leqslant P} e\left(\alpha x^{4}\right), \quad H_{0}(\alpha, \beta)=\prod_{j=2}^{22} h\left(\Lambda_{j}\right), \quad H(\alpha, \beta)=\prod_{j=2}^{s} h\left(\Lambda_{j}\right) .
$$

Then by orthogonality, one has

$$
\mathcal{N}(P) \geqslant \int_{0}^{1} \int_{0}^{1} g\left(\Lambda_{1}\right) H(\alpha, \beta) \mathrm{d} \alpha \mathrm{d} \beta .
$$

In order to define the Hardy-Littlewood dissection underlying our argument, we put $Q=(\log P)^{1 / 100}$, and when $a, b \in \mathbb{Z}$ and $q \in \mathbb{N}$ we define

$$
\mathfrak{N}(q, a, b)=\left\{(\alpha, \beta) \in[0,1)^{2}:|\alpha-a / q| \leqslant Q P^{-4} \text { and }|\beta-b / q| \leqslant Q P^{-4}\right\} .
$$

We then take $\mathfrak{N}$ to be the union of the boxes $\mathfrak{N}(q, a, b)$ with $0 \leqslant a, b \leqslant q \leqslant Q$ and $(q, a, b)=1$. Finally, we put $\mathfrak{n}=[0,1)^{2} \backslash \mathfrak{N}$.

The contribution of the major arcs $\mathfrak{N}$ in this dissection satisfies

$$
\iint_{\mathfrak{N}} g\left(\Lambda_{1}\right) H(\alpha, \beta) \mathrm{d} \alpha \mathrm{d} \beta \gg P^{s-8},
$$

a fact we confirm in $\S 7$. Meanwhile, in $\S 6$ we show that

$$
\iint_{\mathfrak{n}} g\left(\Lambda_{1}\right) H(\alpha, \beta) \mathrm{d} \alpha \mathrm{d} \beta \ll P^{s-8}(\log \log P)^{-1} .
$$

The desired conclusion $\mathcal{N}(P) \gg P^{s-8}$ is immediate from (5.4) and (5.5) on noting that $[0,1)^{2}$ is the disjoint union of $\mathfrak{N}$ and $\mathfrak{n}$.

\section{The Minor ARC TREATMEnT}

The analysis of the minor arc contribution proceeds in two phases, one dominated by the use of Weyl's inequality, and a second in which pruning methods are deployed. We begin preparatory work for the first stage by deriving a consequence of Theorem 2.3. 
Lemma 6.1. One has

$$
\int_{0}^{1} \int_{0}^{1}\left|H_{0}(\alpha, \beta)\right| \mathrm{d} \alpha \mathrm{d} \beta \ll P^{13+\frac{1}{2} \Delta_{10}+\varepsilon} .
$$

Proof. Recall the discussion of the multiplicities associated with $\Lambda_{2}, \ldots, \Lambda_{t}$, and in particular the hypothesis (5.3). By applying [3, Lemma 5], just as in the deduction of [3, equation (4.5)], one finds that

$$
\int_{0}^{1} \int_{0}^{1}\left|H_{0}(\alpha, \beta)\right| \mathrm{d} \alpha \mathrm{d} \beta \ll \int_{0}^{1} \int_{0}^{1} h_{2}^{r_{2}} \ldots h_{t}^{r_{t}} \mathrm{~d} \alpha \mathrm{d} \beta,
$$

where we have abbreviated $\left|h\left(\Lambda_{j}\right)\right|$ to $h_{j}$. Let $\nu$ be a non-negative integer, and suppose that $r_{t-1}=r_{t}+\nu<7$. Then we may apply the argument of the proof of [3, Lemma 6] following equation (4.5) therein to obtain a bound of the shape (6.1), in which $r_{t-1}$ and $r_{t}=r_{t-1}-\nu$ are replaced by $r_{t-1}+1$ and $r_{t}-1$, respectively, or else by $r_{t-1}-\nu-1$ and $r_{t}+\nu+1$. By relabelling if necessary, we thus derive a bound of the shape (6.1), subject to (5.3), wherein either $r_{t}$ is reduced, or else $t$ is reduced. By repeating this process, therefore, we ultimately arrive at the situation in which $t=3$ and $\left(r_{1}, r_{2}, r_{3}\right)=(7,7,7)$. From here the desired estimate follows from (6.1) and Theorem 2.3.

In order to prepare for the pruning process, we introduce a conventional set of one-dimensional major arcs. Define $\mathfrak{M}$ to be the union of the intervals

$$
\mathfrak{M}(q, a)=\left\{\alpha \in[0,1):|q \alpha-a| \leqslant P^{-7 / 2}\right\}
$$

with $0 \leqslant a \leqslant q \leqslant P^{1 / 2}$ and $(a, q)=1$, and put $\mathfrak{m}=[0,1) \backslash \mathfrak{M}$. In addition, when $\lambda, A \in \mathbb{R}$, define the mean value $J(\lambda)=J(\lambda ; A)$ by putting

$$
J(\lambda ; A)=\int_{\mathfrak{M}}\left|g\left(a_{1} \theta\right)\right|^{9 / 4}|h(A \theta+\lambda)|^{4} \mathrm{~d} \theta .
$$

Lemma 6.2. For each $A \in \mathbb{Q} \backslash\{0\}$, one has $\sup _{\lambda \in \mathbb{R}} J(\lambda ; A) \ll P^{9 / 4}$.

Proof. Suppose that $\lambda \in \mathbb{R}$. Write $A=B / S$ with $B \in \mathbb{Z} \backslash\{0\}, S \in \mathbb{N}$ and $(B, S)=1$. We define the modified set of major arcs $\mathfrak{W}$ by putting

$$
\mathfrak{W}=\{\beta \in[0,1): S \beta \in \mathfrak{M}\} .
$$

Then a change of variable yields

$$
J(\lambda)=S \int_{\mathfrak{W}}\left|g\left(a_{1} S \beta\right)\right|^{9 / 4}|h(B \beta+\lambda)|^{4} \mathrm{~d} \beta .
$$

It follows from the definition of $\mathfrak{M}$ that for each $\beta \in \mathfrak{W}$, there exist $c \in \mathbb{Z}$ and $r \in \mathbb{N}$ with $0 \leqslant c \leqslant r \leqslant P^{1 / 2},(c, r)=1$ and $|S \beta r-c| \leqslant P^{-7 / 2}$. Thus there exist $a \in \mathbb{Z}$ and $q \in \mathbb{N}$ with $0 \leqslant a \leqslant q \leqslant S P^{1 / 2},(a, q)=1$ and $|q \beta-a| \leqslant P^{-7 / 2}$. Next, we define $\kappa(q)$ to be the multiplicative function defined for $q \in \mathbb{N}$ by taking, for prime numbers $p$ and non-negative integers $l$,

$$
\kappa\left(p^{4 l}\right)=p^{-l}, \quad \kappa\left(p^{4 l+1}\right)=4 p^{-l-1 / 2}, \quad \kappa\left(p^{4 l+2}\right)=p^{-l-1}, \quad \kappa\left(p^{4 l+3}\right)=p^{-l-1} .
$$


Then as a consequence of [13, Theorem 4.1 and Lemmata 4.3, 4.4 and 6.2],

$$
\begin{aligned}
g\left(a_{1} S \beta\right) & \ll \kappa(q) P\left(1+P^{4}|\beta-a / q|\right)^{-1}+q^{1 / 2+\varepsilon} \\
& \ll \kappa(q) P\left(1+P^{4}|\beta-a / q|\right)^{-1 / 2} .
\end{aligned}
$$

We therefore deduce from (6.3) that

$$
J(\lambda) \ll \sum_{1 \leqslant q \leqslant S P^{1 / 2}}(P \kappa(q))^{9 / 4} \sum_{a=1}^{q} \int_{-\infty}^{\infty} \frac{|h(B(a / q+\gamma)+\lambda)|^{4}}{\left(1+P^{4}|\gamma|\right)^{9 / 8}} \mathrm{~d} \gamma .
$$

By orthogonality, we find that

$$
\sum_{a=1}^{q}|h(B(a / q+\gamma)+\lambda)|^{4} \leqslant q \sum_{\substack{1 \leqslant x_{1}, \ldots, x_{4} \leqslant P \\ q \mid B\left(x_{1}^{4}+x_{2}^{4}-x_{3}^{4}-x_{4}^{4}\right)}} 1 \leqslant|B|^{4}\left(P q^{-1}+1\right)^{4} q \rho(q),
$$

where $\rho(q)$ denotes the number of solutions of the congruence

$$
x_{1}^{4}+x_{2}^{4} \equiv x_{3}^{4}+x_{4}^{4}(\bmod q) \text {, }
$$

with $1 \leqslant x_{i} \leqslant q(1 \leqslant i \leqslant 4)$. The argument of [14] leading to equation (5.8) of that paper shows that

$$
q \rho(q) \ll q^{4} \sum_{r \mid q} r \kappa(r)^{4} .
$$

Hence, on substituting (6.5) into (6.4), we obtain

$$
J(\lambda) \ll P^{25 / 4} \sum_{1 \leqslant q \leqslant S P^{1 / 2}} \kappa(q)^{9 / 4} \sum_{r \mid q} r \kappa(r)^{4} \int_{-\infty}^{\infty}\left(1+P^{4}|\gamma|\right)^{-9 / 8} \mathrm{~d} \gamma .
$$

Observe that $\kappa(q)^{9 / 4} \leqslant q^{-1 / 20} \kappa(q)^{2}$, and hence the argument completing the proof of [14, Lemma 5.4] shows that for a suitable positive constant $C$, one has

$$
J(\lambda) \ll P^{9 / 4} \sum_{1 \leqslant q \leqslant P} \kappa(q)^{9 / 4} \sum_{r \mid q} r \kappa(r)^{4} \ll P^{9 / 4} \prod_{p}\left(1+C \sum_{h=1}^{\infty} p^{-1-h / 20}\right) .
$$

Thus we obtain $J(\lambda) \ll P^{9 / 4}$, and the proof of the lemma is complete.

When $\mathfrak{B} \subseteq[0,1)^{2}$ is measurable, define the auxiliary mean value

$$
U(\mathfrak{B})=\iint_{\mathfrak{B}}\left|g\left(\Lambda_{1}\right) H_{0}(\alpha, \beta)\right| \mathrm{d} \alpha \mathrm{d} \beta .
$$

Lemma 6.3. One has $U(\mathfrak{n}) \ll P^{14}(\log \log P)^{-1}$.

Proof. Consider the auxiliary sets

$$
\mathfrak{e}=\{(\alpha, \beta) \in \mathfrak{n}: \alpha \in \mathfrak{m}\} \quad \text { and } \quad \mathfrak{E}=\{(\alpha, \beta) \in \mathfrak{n}: \alpha \in \mathfrak{M}\} .
$$

The treatment of the set $\mathfrak{e}$ is straightforward. On recalling that $\Lambda_{1}=a_{1} \alpha$, one finds via an enhanced version of Weyl's inequality (see [11, Lemma 3]) that

$$
\sup _{(\alpha, \beta) \in \mathfrak{e}}\left|g\left(\Lambda_{1}\right)\right|=\sup _{\alpha \in \mathfrak{m}}\left|g\left(a_{1} \alpha\right)\right| \ll P^{7 / 8+\varepsilon} \text {. }
$$


Then from Lemma 6.1, one deduces that

$$
U(\mathfrak{e}) \ll P^{7 / 8+\varepsilon} \int_{0}^{1} \int_{0}^{1}\left|H_{0}(\alpha, \beta)\right| \mathrm{d} \alpha \mathrm{d} \beta \ll P^{\frac{111}{8}+\frac{1}{2} \Delta_{10}+\varepsilon} \ll P^{14} Q^{-1} .
$$

We turn next to the complementary set $\mathfrak{E}$, handling this via Lemma 6.2. By applying the argument underlying the proof of Lemma 6.1, as in the discussion following the statement of [3, Lemma 10], one finds that for some indices $l, m, n$ with $2 \leqslant l<m<n \leqslant 22$, one has

$$
U(\mathfrak{E}) \ll \iint_{\mathfrak{E}} g_{1} h_{l}^{7} h_{m}^{7} h_{n}^{7} \mathrm{~d} \alpha \mathrm{d} \beta .
$$

Here, we have abbreviated $\left|g\left(\Lambda_{1}\right)\right|$ to $g_{1}$. By relabelling variables if necessary, there is no loss of generality in supposing that $(l, m, n)=(2,3,4)$ and that $\Lambda_{3}$ and $\Lambda_{4}$ are each pairwise linearly independent of $\Lambda_{1}$. Recall also that $\Lambda_{2}, \Lambda_{3}, \Lambda_{4}$ are assumed to be pairwise linearly independent. Define

$$
U_{i j}=\iint_{\mathfrak{E}} g_{1}^{9 / 4} h_{i}^{4} h_{j}^{12} \mathrm{~d} \alpha \mathrm{d} \beta \quad \text { and } \quad V_{i j}=\iint_{\mathfrak{E}} h_{i}^{12} h_{j}^{12} \mathrm{~d} \alpha \mathrm{d} \beta .
$$

Then an application of Hölder's inequality yields the bound

$$
U(\mathfrak{E}) \ll\left(\sup _{(\alpha, \beta) \in \mathfrak{E}}\left|h\left(\Lambda_{2}\right) h\left(\Lambda_{3}\right) h\left(\Lambda_{4}\right)\right|\right)^{5 / 27}\left(U_{23} U_{24}\right)^{2 / 9}\left(V_{23} V_{24}\right)^{17 / 81} V_{34}^{11 / 81} .
$$

Let $(i, j)$ be either $(2,3)$ or $(2,4)$. Recall that $\Lambda_{1}=a_{1} \alpha$, and change variables from $\beta$ to $\gamma$ via the linear transformation $a_{j} \alpha+b_{j} \beta=b_{j} \gamma$. Note here that since $\Lambda_{1}$ and $\Lambda_{j}$ are inequivalent, then necessarily $b_{j} \neq 0$. Write $A=a_{i}-b_{i} a_{j} / b_{j}$ and recall (6.2). Then in view of the definition of $\mathfrak{E}$, we may make use of the periodicity of the integrand to deduce that

$$
U_{i j} \leqslant \int_{0}^{1} \int_{\mathfrak{M}}\left|g\left(\Lambda_{1}\right)\right|^{9 / 4}\left|h\left(A \alpha+b_{i} \gamma\right)\right|^{4}\left|h\left(b_{j} \gamma\right)\right|^{12} \mathrm{~d} \alpha \mathrm{d} \gamma \leqslant W \sup _{\lambda \in \mathbb{R}} J(\lambda ; A),
$$

where

$$
W=\int_{0}^{1}\left|h\left(b_{j} \gamma\right)\right|^{12} \mathrm{~d} \gamma
$$

An application of Lemma 2.1 shows, via a change of variable, that $W=O\left(P^{8}\right)$, and so we deduce from Lemma 6.2 that $U_{23} U_{24} \ll\left(P^{8}\right)^{2}\left(P^{9 / 4}\right)^{2}=P^{41 / 2}$. Since $\Lambda_{2}, \Lambda_{3}, \Lambda_{4}$ are pairwise linearly independent, when $2 \leqslant i<j \leqslant 4$ further changes of variable lead from Lemma 2.1 to the estimate

$$
V_{i j} \ll \int_{0}^{1} \int_{0}^{1}\left|h\left(\theta_{1}\right) h\left(\theta_{2}\right)\right|^{12} \mathrm{~d} \theta_{1} \mathrm{~d} \theta_{2}=\left(\int_{0}^{1}|h(\theta)|^{12} \mathrm{~d} \theta\right)^{2} \ll\left(P^{8}\right)^{2} .
$$

By substituting these estimates into (6.7), we deduce thus far that

$$
U(\mathfrak{E}) \ll P^{14}\left(P^{-3} \sup _{(\alpha, \beta) \in \mathfrak{E}}\left|h\left(\Lambda_{2}\right) h\left(\Lambda_{3}\right) h\left(\Lambda_{4}\right)\right|\right)^{5 / 27} .
$$

Our final task is to bound the second factor on the right hand side of (6.8). When $\theta$ is a real number with $|h(\theta)| \geqslant P Q^{-1 / 100}$, it follows from [15, Lemma $2.1]$ that there exist $a \in \mathbb{Z}$ and $q \in \mathbb{N}$ with $1 \leqslant q \leqslant Q^{1 / 10},(a, q)=1$ and 
$|q \theta-a| \leqslant Q^{1 / 10} P^{-4}$. Consequently, if $\Lambda_{k}$ and $\Lambda_{l}$ are inequivalent linear forms, and $h_{k} h_{l} \geqslant P^{2} Q^{-1 / 100}$, then for $\sigma=k, l$ there exist integers $d_{\sigma}$ and $q_{\sigma}$ with

$$
1 \leqslant q_{\sigma} \leqslant Q^{1 / 10}, \quad\left(d_{\sigma}, q_{\sigma}\right)=1 \quad \text { and } \quad\left|\Lambda_{\sigma}-d_{\sigma} / q_{\sigma}\right| \leqslant q_{\sigma}^{-1} Q^{1 / 10} P^{-4} .
$$

From here it follows as in the proof of [3, Lemma 10] that $(\alpha, \beta) \in \mathfrak{N}$. Thus

$$
\sup _{(\alpha, \beta) \in \mathfrak{n}}\left|h\left(\Lambda_{k}\right) h\left(\Lambda_{l}\right)\right| \ll P^{2} Q^{-1 / 100},
$$

whence we obtain the estimate

$$
\sup _{(\alpha, \beta) \in \mathfrak{E}}\left|h\left(\Lambda_{2}\right) h\left(\Lambda_{3}\right) h\left(\Lambda_{4}\right)\right| \ll P^{3} Q^{-1 / 100} .
$$

On substituting into (6.8), we conclude that $U(\mathfrak{E}) \ll P^{14} Q^{-1 / 600}$. The conclusion of the lemma therefore follows from (6.6) on recalling that $\mathfrak{n}=\mathfrak{e} \cup \mathfrak{E}$.

A trivial estimate for the generating function $h(\theta)$ now leads from the conclusion of Lemma 6.3 to the estimate (5.5) by means of the relation

$$
\iint_{\mathfrak{n}} g\left(\Lambda_{1}\right) H(\alpha, \beta) \mathrm{d} \alpha \mathrm{d} \beta \ll P^{s-22} U(\mathfrak{n}) .
$$

\section{THE MAJOR ARCS ANALYSIS}

Not only is the analysis of the major arcs largely standard, but it is also very similar to the work in [3, §7]. A brief sketch of the analysis therefore suffices on the present occasion. We begin with some additional notation. Define

$$
\begin{gathered}
S(q, a)=\sum_{r=1}^{q} e\left(a r^{4} / q\right), \quad T(q, c, d)=q^{-s} \prod_{j=1}^{s} S\left(q, a_{j} c+b_{j} d\right), \\
\mathfrak{A}(q)=\sum_{\substack{a=1 \\
(a, b, q)=1}}^{q} \sum_{\substack{b=1 \\
(a)}}^{q}(q, a, b) \text { and } \mathfrak{S}(X)=\sum_{1 \leqslant q \leqslant Q} \mathfrak{A}(q) .
\end{gathered}
$$

Also, with $\lambda_{j}$ as shorthand for $a_{j} \xi+b_{j} \zeta$, put

$$
v(\theta)=\int_{0}^{P} e\left(\theta \gamma^{4}\right) \mathrm{d} \gamma, \quad w(\theta)=\int_{\delta P}^{P} e\left(\theta \gamma^{4}\right) \mathrm{d} \gamma, \quad V(\xi, \zeta)=w\left(a_{1} \xi\right) \prod_{j=2}^{s} v\left(\lambda_{j}\right),
$$

and writing $\mathcal{B}(X)=\left[-X P^{-4}, X P^{-4}\right]^{2}$, define

$$
\mathfrak{J}(X)=\iint_{\mathcal{B}(X)} V(\xi, \zeta) \mathrm{d} \xi \mathrm{d} \zeta .
$$

Recall that $\Lambda_{1}=a_{1} \alpha$. Then, as adapted to the current context, the argument leading to [3, equation (7.5)] shows that there is a positive number $\rho$ having the property that whenever $(\alpha, \beta) \in \mathfrak{N}(q, a, b) \subseteq \mathfrak{N}$, one has

$$
g\left(\Lambda_{1}\right) H(\alpha, \beta)-\rho T(q, a, b) V(\alpha-a / q, \beta-b / q) \ll P^{s}(\log P)^{-1 / 2} .
$$

Integrating over $\mathfrak{N}$, we infer that

$$
\iint_{\mathfrak{N}} g\left(\Lambda_{1}\right) H(\alpha, \beta) \mathrm{d} \alpha \mathrm{d} \beta=\rho \mathfrak{S}(Q) \mathfrak{J}(Q)+O\left(P^{s-8}(\log P)^{-1 / 4}\right) .
$$


Lemma 7.1. Under the hypotheses of Theorem 1.1, the limit $\mathfrak{S}=\lim _{X \rightarrow \infty} \mathfrak{S}(X)$ exists, and one has $\mathfrak{S}-\mathfrak{S}(X) \ll X^{-1}$. When the pair of equations (1.1) has a non-singular $p$-adic solution for all primes $p$, moreover, one has $\mathfrak{S} \gg 1$.

Proof. This lemma is an adaptation of [3, Lemma 12] to our needs. We establish the estimate $\mathfrak{A}(q)=O\left(q^{-2}\right)$ to replace the cognate bound [3, equation (7.14)]. Once this is confirmed, all the conclusions drawn in the lemma follow just as in the aforementioned work [3], and thus we may omit the details. We immitate the argument on [3, page 890] to establish the aforementioned bound. Note first that we may suppose that whenever $(c, d) \in \mathbb{Z}^{2} \backslash\{(0,0)\}$, then the linear form $c L_{1}(\boldsymbol{\theta})+d L_{2}(\boldsymbol{\theta})$ contains at least $s-7$ non-zero coefficients. Let $u_{j}=\left(q, c a_{j}+d b_{j}\right)$ and apply [13, Theorem 4.2] to infer that

$$
T(q, c, d) \ll q^{-11 / 2}\left(u_{1} u_{2} \ldots u_{22}\right)^{1 / 4} .
$$

Here, we have used the prearrangement of indices and removed dependence on potential indices with $22<j \leqslant s$ by the use of trivial estimates. Recall now the multiplicities $r_{1}, \ldots, r_{t}$ associated to the equivalence classes of the forms $\Lambda_{j}$. Following the analysis on [3, page 890], one finds that there is a natural number $\Delta$ depending only on $a_{j}, b_{j}(1 \leqslant j \leqslant s)$ such that

$$
\mathfrak{A}(q) \ll q^{-7 / 2} \sum_{\substack{v_{1}, \ldots, v_{t} \\ v_{1} v_{2} \ldots v_{t} \mid \Delta q}} v_{1}^{\left(r_{1}-4\right) / 4} \ldots v_{t}^{\left(r_{t}-4\right) / 4} .
$$

The upper bound $r_{l} \leqslant 7$ therefore leads to the estimate $\mathfrak{A}(q) \ll q^{\varepsilon-11 / 4}$, so that in view of our earlier comments, the proof of the lemma is complete.

Lemma 7.2. Under the hypotheses of Theorem 1.1, the limit $\mathfrak{J}=\lim _{X \rightarrow \infty} \mathfrak{J}(X)$ exists, and one has $\mathfrak{J}-\mathfrak{J}(X) \ll P^{s-8} X^{-1}$. When the pair of equations (1.1) has a non-singular real solution, moreover, one has $\mathfrak{J} \gg P^{s-8}$.

Proof. Write $\widehat{\mathcal{B}}(X)$ for $\mathbb{R}^{2} \backslash \mathcal{B}(X)$, and recall the prearrangement of indices introduced in $\S 5$. Then a direct modification of the argument on [3, page 891] confirms that, for a suitable positive number $\Theta=\Theta(\mathbf{a}, \mathbf{b})$, one has

$$
\iint_{\widehat{\mathcal{B}}(X)}\left|v\left(\lambda_{2}\right) \ldots v\left(\lambda_{22}\right)\right| \mathrm{d} \xi \mathrm{d} \zeta \ll P^{21} \iint_{\widehat{\mathcal{B}}(\Theta X)}\left(1+P^{4}|\xi|\right)^{-21 / 8}\left(1+P^{4}|\zeta|\right)^{-21 / 8} \mathrm{~d} \xi \mathrm{d} \zeta .
$$

By applying trivial bounds for $w\left(a_{1} \xi\right)$ and the additional factors $v\left(\lambda_{j}\right)$ for $j>22$, we therefore conclude that

$$
\iint_{\mathbb{R}^{2} \backslash \mathcal{B}(X)} V(\xi, \zeta) \mathrm{d} \xi \mathrm{d} \zeta \ll P^{s-21}\left(P^{13} X^{-1}\right) \ll P^{s-8} X^{-1} .
$$

This bound replaces [3, equation (7.18)], and the lemma now follows in the same manner as Lemma 13 was proved in [3]. 
The conclusions of Lemmata 7.1 and 7.2 now combine with the asymptotic formula (7.1) to deliver the relation

$$
\iint_{\mathfrak{N}} g\left(\Lambda_{1}\right) H(\alpha, \beta) \mathrm{d} \alpha \mathrm{d} \beta=\rho \mathfrak{S} \mathfrak{J}+O\left(P^{s-8} Q^{-1}\right) \gg P^{s-8},
$$

thereby confirming the lower bound (5.4). In view of the discussion concluding $\S 5$, the lower bound $\mathcal{N}(P) \gg P^{s-8}$ that establishes Theorem 1.1 now follows.

\section{REFERENCES}

[1] J. Brüdern and R. J. Cook, On simultaneous diagonal equations and inequalities, Acta Arith. 62 (1992), no. 2, 125-149.

[2] J. Brüdern and T. D. Wooley, On Waring's problem: two cubes and seven biquadrates, Tsukuba J. Math. 24 (2000), no. 2, 387-417.

[3] J. Brüdern and T. D. Wooley, The Hasse principle for pairs of diagonal cubic forms, Ann. of Math. (2) 166 (2007), no. 3, 865-895.

[4] J. Brüdern and T. D. Wooley, Asymptotic formulae for pairs of diagonal cubic equations, Canad. J. Math. 63 (2011), no. 1, 38-54.

[5] J. Brüdern and T. D. Wooley, The Hasse principle for systems of diagonal cubic forms, submitted, arXiv:1304.5165.

[6] R. J. Cook, A note on a lemma of Hua, Quart. J. Math. Oxford Ser. (2) 23 (1972), $287-288$.

[7] H. Dym and H. P. McKean, Fourier series and integrals, Academic Press, New YorkLondon, 1972.

[8] H. Godinho, A pair of additive quartic forms, Ph.D. thesis, University of Michigan, 1992.

[9] K. Kawada and T. D. Wooley, Davenport's method and slim exceptional sets: the asymptotic formulae in Waring's problem, Mathematika 56 (2010), no. 2, 305-321.

[10] S. Poehler, Two additive quartic forms, Ph.D. thesis, Universität Stuttgart, 2007; available at http://elib.uni-stuttgart.de/opus/volltexte/2007/3182/

[11] R. C. Vaughan, On Waring's problem for smaller exponents. II, Mathematika 33 (1986), no. $1,6-22$.

[12] R. C. Vaughan, A new iterative method in Waring's problem, Acta Math. 162 (1989), no. $1-2,1-71$.

[13] R. C. Vaughan, The Hardy-Littlewood method, 2nd edition, Cambridge University Press, Cambridge, 1997.

[14] R. C. Vaughan and T. D. Wooley, Further improvements in Waring's problem, IV: Higher powers, Acta Arith. 94 (2000), no. 3, 203-285.

[15] T. D. Wooley, On Diophantine inequalities: Freeman's asymptotic formulae, Proceedings of the session in analytic number theory and Diophantine equations (Bonn, January-June, 2002), Bonn 2003, Edited by D. R. Heath-Brown and B. Z. Moroz, Bonner Mathematische Schriften, Nr. 360, Article 30, 32pp.

[16] A. Zygmund, Trigonometric series, Vol. I, Third edition, Cambridge University Press, Cambridge, 2002.

Mathematisches Institut, Bunsenstrasse 3-5, D-37073 Göttingen, Germany E-mail address: bruedern@uni-math.gwdg.de

School of Mathematics, University of Bristol, University Walk, Clifton, Bristol BS8 1TW, United Kingdom

E-mail address: matdw@bristol.ac.uk 